\title{
The Effect of Postural Correction and Visual Feedback on Muscle Activity and Head Position Change During Overhead Arm Lift Test in Subjects with Forward Head Posture
}

\author{
Liwen Xu, Byoungha Hwang, Teaho Kim \\ Department of Physical Therapy, College of Rehabilitation Science, Daegu University, Gyeongsan, Korea
}

\begin{abstract}
Purpose: This study aimed to investigate the immediate effects of posture correction and real-time visual feedback using a video display on muscle activity and change of head position during overhead arm lift test in individuals with forward head posture.

Methods: Fifteen subjects with forward head posture and fifteen normal subjects who volunteered were included in this study. During both groups performed the overhead arm lift test, the muscle activity of the upper trapezius, serratus anterior, sternocleidomastoid, and lower trapezius muscle were measured using electromyography, and head position change was measured using photographs. Then, forward head posture group was asked to perform overhead arm lift test again after posture correction and real-time visual feedback using a video display respectively. One-way analysis of variance (ANOVA) was used to analyze four conditions: pre-test, posture correction, real-time visual feedback, and the control group.

Results: The upper trapezius and lower trapezius muscle activity significantly decreased posture correction, real-time visual feedback, and control group than pre-test of forward head posture group $(p<0.05)$. The sternocleidomastoid muscle significantly decreased realtime visual feedback and control group than pre-test of forward head posture group. Head position change significantly decreased three conditions than pre-test of forward head posture group and real-time visual feedback and control group significantly decreased than posture correction.

Conclusion: This study recommend for maintaining cervical stability during the overhead arm lift test, postural control using real-time visual feedback is more effective in subjects with forward head posture.
\end{abstract}

Keywords: Forward head posture, Overhead arm lift test, Real-time visual feedback

\section{INTRODUCTION}

In the current age, people tend to contract their shoulders and neck muscles for long periods of time to maintain a seated posture before computer screens. This can result in accumulated fatigue and mechanical pain caused by incorrect posture. ${ }^{1}$

Neck pain is frequently reported in the workplace and is environment-related. Computer use affects the musculoskeletal system; looking into a monitor below the line of sight for extended time periods makes the head move forward, which produces an exaggerated anterior curve in the lower cervical vertebrae and an exaggerated posterior curve in the upper thoracic vertebrae to maintain balance.

Received May 10, 2019 Revised Jun 21, 2019

Accepted Jun 29, 2019

Corresponding author Teaho Kim

E-mail ptkimth@daegu.ac.kr
This is known as forward head posture. ${ }^{2,3}$ Chiu et al. ${ }^{4}$ found that approximately $60 \%$ of individuals with neck and shoulder pain exhibited forward head posture.

In a normal head position, the tragus and acromial angle are in a vertical position, but in forward head posture, a horizontal distance of over $5 \mathrm{~cm}$ extends between the tragus and the acromial angle, accompanied by bending in the lower cervical area and hyperextension in the upper cervical area. ${ }^{5}$

Forward head posture leads to lengthening and weakness in the anterior cervical muscles as well as shortening in the posterior cervical muscles. ${ }^{6}$ It will also shorten the upper trapezius and sternocleidomastoid and weaken the serratus anterior. ${ }^{7}$ Further, it may af-

Copylight (C)2019 The Korean Society of Physical Therapy

This is an Open Access article distribute under the terms of the Creative Commons Attribution Non-commercial License (Http:// creativecommons.org/license/by-nc/4.o.) which permits unrestricted non-commercial use, distribution, and reproduction in any medium, provided the original work is properly cited. 
fect not only the neck but also the thoracic spine and the scapulae, possibly causing overall imbalance in the musculoskeletal system. ${ }^{8}$ The neck muscle imbalance caused by forward head posture creates an abnormal scapula tilt and cause a condition in the lower trapezius and serratus anterior known as rounded shoulder.

Postural correction aims to reduce the static load on surrounding muscles, and the correction of cervical posture is often recommended for the treatment and prevention of chronic neck and shoulder pain. ${ }^{10}$ To prevent shoulder and neck pain associated with abnormal neck posture, many researchers have stressed the importance of maintaining a neutral head posture during arm movement and functional activity. ${ }^{11}$

Comerford and Mottram ${ }^{12}$ developed the overhead arm lift test to assess a subject's ability to actively dissociate and control low cervical flexion and move the shoulders through overhead flexion. They suggested patients should be able to keep the head neutral while actively flexing the shoulders and lifting the arms to a 180-degree flexion. An ideal posture in the overhead arm lift test is achievable if the neck is sufficiently stable. However, as the human field of vision is limited, it is impossible to visually self-monitor head posture during this treatment.

Real-time visual feedback has been used during biomechanical analyses for such purposes as helping participants adjust their mechanics to avoid injury and match certain protocols and to provide information about force production or muscle activity. ${ }^{13}$ Weon et al. ${ }^{14}$ have noted that real-time visual feedback can be recommended for increasing the activity of the upper trapezius and SA in people with scapular winging through shoulder flexion.

However, few studies have investigated the effects of real-time visual feedback on muscle activity and head position change in the FHP during the overhead arm lift test. The purpose of this study was to determine whether real-time visual feedback would alter the electromyographic activity of the upper trapezius, lower trapezius, serratus anterior, and sternocleidomastoid or the head position change during the overhead arm lift test in subjects with forward head posture.

\section{METHODS}

\section{Subjects}

This study was conducted with 30 participants, with 15 in the for-
Table 1. General characteristics of subjects

\begin{tabular}{lccc}
\hline & FHG $(n=15)$ & CG $(n=15)$ & $p$ \\
\hline Gender & & & \\
Male & 9 & 6 & 0.28 \\
Female & 6 & 9 & \\
Age (year) & $21.60 \pm 1.63^{+}$ & $20.87 \pm 0.99$ & 0.15 \\
Body height $(\mathrm{cm})$ & $169.53 \pm 8.11$ & $165.67 \pm 8.20$ & 0.21 \\
Body weight $(\mathrm{kg})$ & $61.93 \pm 11.67$ & $55.40 \pm 9.10$ & 0.10 \\
CVA $\left(^{\circ}\right)$ & $50.16 \pm 2.16$ & $56.43 \pm 2.27$ & $0.00^{*}$
\end{tabular}

FHG: forward head posture group, CG: control group, CVA: craniovertebral angle.

${ }^{+}$Mean $\pm S D,{ }^{*} p<0.05$

ward head posture group and 15 in the control group. If the CVA angle was less than $53^{\circ}$, was criteria in the forward head posture group. Subjects were recruited from Daegu University in Gyeongsan, Korea. Prior to participation, all participants received an explanation about the study and signed the university-approved human subjects' consent form. This study was approved by the Daegu University Institutional Review Board. The demographic and clinical characteristics of these patients are given in Table 1.

\section{Experimental methods}

\section{1) Experimental procedure}

All subjects had surface electrodes placed on the right side before the beginning of the experiment. Both groups were performed the overhead arm lift test. The forward head posture group then underwent two different intervention conditions through this test. The first was to sit with correct posture on the vertical line during the overhead arm lift test. In the second, subjects were asked to perform an overhead lift test while watching a real-time visual feedback monitor. All measurements were repeated three times to produce average values.

\section{(1) Measurement of forward head and head position changes}

Lateral photographs of subjects in both groups were taken to facilitate measurement of the CVA and head position changes. Markers were attached to subjects' C7 vertebrae and tragi. The CVA is defined as the angle between a horizontal line passing through the $\mathrm{C} 7$ vertebra and a line extending from the tragus of the ear to the $\mathrm{C} 7$ vertebra. ${ }^{15}$ If the CVA angle was less than $53^{\circ}$, the subject was placed in the experimental group. ${ }^{16}$ Changes in head position were measured according to the distance between a vertical line passing 
through the tragus marker and the vertical line at the forefront of a grid line. ${ }^{17}$

\section{(2) Surface electromyography}

Data relating to muscle activity during the overhead arm lift test were collected via a wireless EMG system (TeleMyo DTS, Noraxon Inc., Scottsdale) using silver-silver chloride dual surface electrodes. The positioning of the surface electrodes on each muscle was arranged in congruence with previous studies. ${ }^{18}$ Before the electrodes were attached, subjects' skin was cleaned with alcohol to reduce electrical impedance.

EMG data were normalized using the maximal voluntary isometric contractions (MVIC) of the upper trapezius, lower trapezius, serratus anterior, and sternocleidomastoid separately. The measurement positions for the MVIC were chosen according to a study by

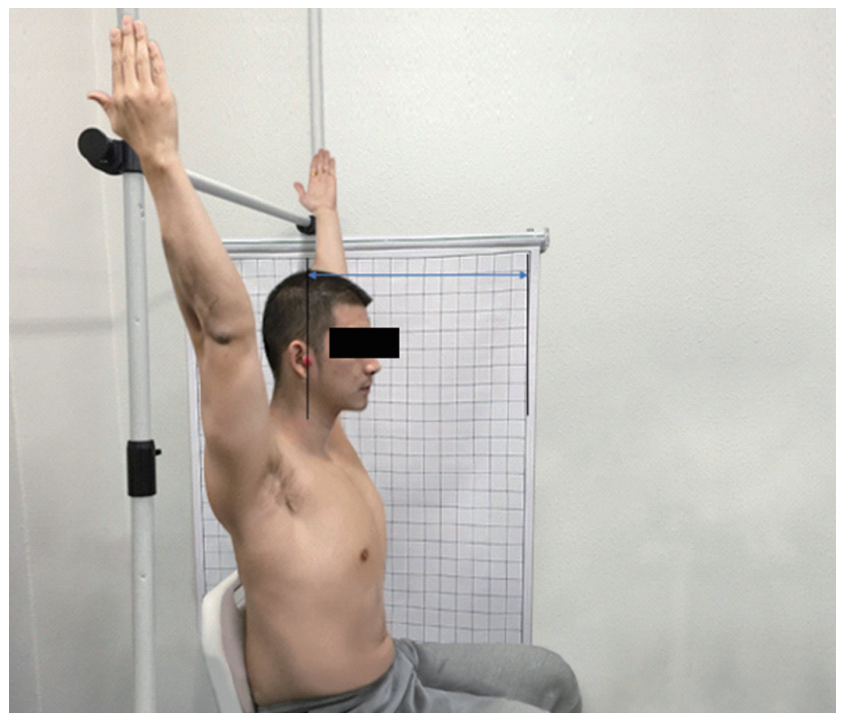

Figure 1. Overhead arm lift test and head position change.
Kendall. ${ }^{7}$ MVIC values reflected the average RMS after three trials. EMG data were used for the three seconds of keep the end range for five seconds and expressed as a percentage of MVIC (\%MVIC).

\section{(3) Overhead arm lift test}

All subjects sat in a chair with a backrest and looked straight ahead. The examiner instructed the subjects to sit comfortably, lift their arms to the top of their overhead, up to a horizontal bar and holding the posture for 5 seconds and then lower them sideways. At this point, the head should maintain in a neutral position while the arms are fully lifted (at a $180^{\circ}$ flexion) and lowered. ${ }^{12}$

To measure head position changes during the overhead arm lift test, a photograph was taken with a lateral view before and after the test Image J software was used for photographic analysis (Figure 1).

\section{2) Intervention methods}

\section{(1) Posture correction}

In bodies with excellent posture, the line in the sagittal plane extends through the ear lobe, the tip of the shoulder, the center of the hip and the knee, and slightly anterior to the external malleolus. ${ }^{19}$ Testers adjusted subjects' heads to a corrective position manually, and a vertical line was used as the baseline (Figure 2).

\section{(2) Real-time visual feedback correction}

The subjects were informed about a corrective head position. They were then required to maintain this posture and perform end-range shoulder flexion for 5 seconds while their head posture was observed through a computer monitor (Figure 2). A video camera (DCR-SR68E, Sony, Tokyo, Japan) input into the computer monitor provided real-time visual posture feedback to the forward head pos-
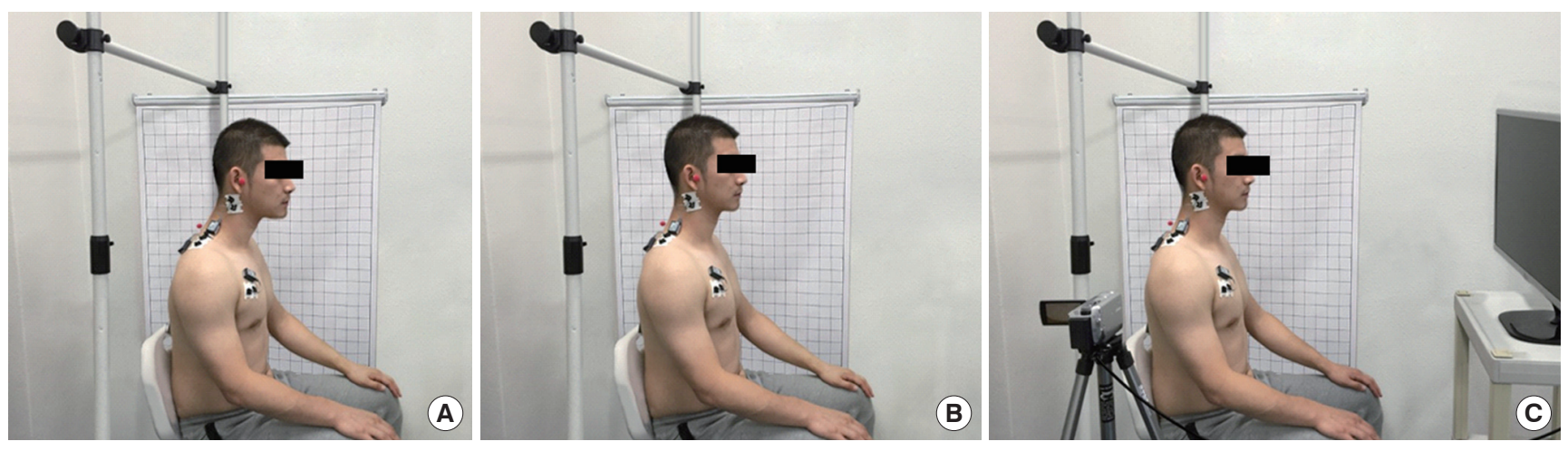

Figure 2. Posture correction and real-time visual feedback. (A) Forward head posture, (B) posture correction, (C) real-time visual feedback. 
ture group. The video camera was set at the height of the subjects' acromia to expose the part of the head and the shoulders entirely. The monitor was set at the height of each subject's sightline.

\section{3) Statistical analysis}

The Kolmogorov-Smirnov test was used for a normal distribution. One-way analysis of variance (ANOVA) was used to analyze four conditions: pre-test, posture correction, real-time visual feedback, and the control group. The post-hoc analysis was performed to investigate significant differences between each of the four conditions by LSD. Statistical analyses were performed using SPSS for Windows (version 20.0), and the statistical significance level was set at $\mathrm{p}<0.05$.

\section{RESULTS}

The upper trapezius and lower trapezius muscle activity significantly decreased posture correction, real-time visual feedback, and control group than pre-test of forward head posture group $(\mathrm{p}<0.05$, Table 2)(Figure 3). The sternocleidomastoid muscle significantly decreased real-time visual feedback and control group than pre-test of forward head posture group $(\mathrm{p}<0.05$, Table 2$)$ (Figure 3).

Serratus anterior muscle activity was not significantly between all conditions ( $p>0.05$, Table 2)(Figure 3).

Head position change significantly decreased three conditions than pre-test of forward head posture group and real-time visual feedback and control group significantly decreased than posture correction $(\mathrm{p}<0.05$, Table 3)(Figure 3).

\section{DISCUSSION}

This study measured both forward head posture subjects and control subjects to identify the differences in muscle activity and head position changes between the two groups through the overhead arm lift test. Only the forward head posture group was measured again after posture correction and real-time visual feedback was provided, as this study focused on subjects with FHP. The upper trapezius muscle activity significantly decreased posture correction

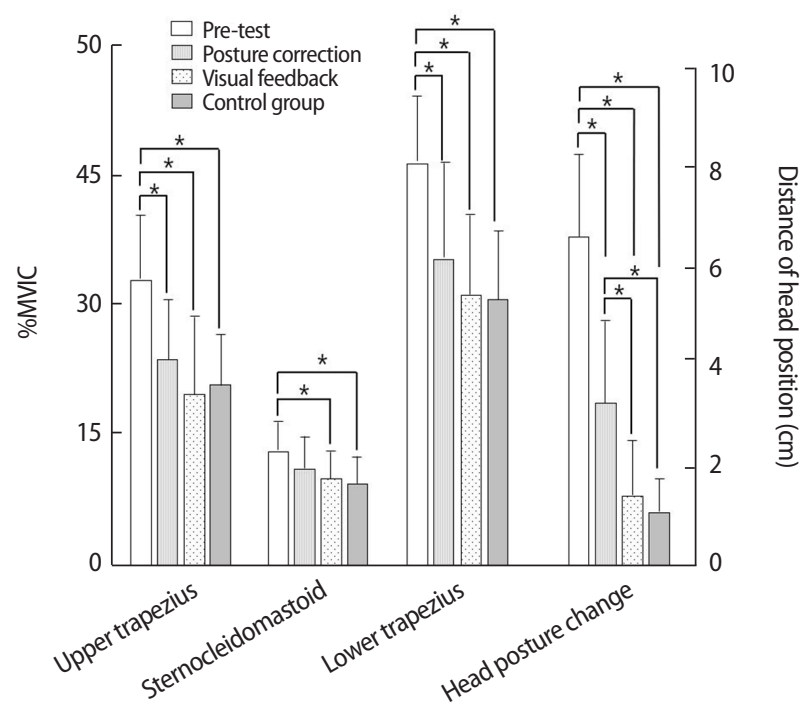

Figure 3. Comparison of muscle activity and head position change $\left({ }^{*} p<0.05\right)$.

Table 2. Comparison of muscle activity in four conditions

(unit: \%MVIC)

\begin{tabular}{|c|c|c|c|c|c|c|}
\hline & & FHG $(n=15)$ & & $C F(n-15)$ & 5 & 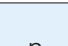 \\
\hline & Pre & PC & VF & $(3)(11-15)$ & $r$ & p \\
\hline$\overline{\text { UT }}$ & $32.46 \pm 7.63^{+}$ & $23.33 \pm 7.36$ & $19.05 \pm 8.86$ & $20.36 \pm 6.81$ & 8.22 & $0.00^{*}$ \\
\hline SA & $26.64 \pm 6.14$ & $31.76 \pm 7.45$ & $34.16 \pm 10.04$ & $35.24 \pm 13.40$ & 2.35 & 0.08 \\
\hline SCM & $12.79 \pm 4.01$ & $10.54 \pm 4.43$ & $9.06 \pm 3.56$ & $8.68 \pm 3.00$ & 3.61 & $0.02^{*}$ \\
\hline LT & $45.64 \pm 10.51$ & $35.61 \pm 10.49$ & $31.77 \pm 11.51$ & $30.03 \pm 8.53$ & 6.88 & $0.00^{*}$ \\
\hline
\end{tabular}

FHG: forward head posture group, CG: control group, Pre: pre-test, PC: posture correction, VF: visual feedback, UT: upper trapezius, SA: serratus anterior, SCM: sternocleidomastoid, LT: lower trapezius.

${ }^{+}$Mean $\pm S D,{ }^{*} p<0.05$.

Table 3. Comparison of head position change between two group and intervention

(unit: $\mathrm{cm}$ )

\begin{tabular}{|c|c|c|c|c|c|}
\hline \multicolumn{3}{|c|}{ FHG $(n=15)$} & \multirow{2}{*}{$C G(n=15)$} & \multirow{2}{*}{$\mathrm{F}$} & \multirow{2}{*}{$p$} \\
\hline Pre & PC & VF & & & \\
\hline $6.65 \pm 2.08^{+}$ & $3.25 \pm 1.88$ & $1.57 \pm 1.16$ & $1.19 \pm 0.60$ & 38.75 & $0.00^{*}$ \\
\hline
\end{tabular}

FHG: forward head posture group, CG: control group, Pre: pre-test, PC: posture correction, VF: visual feedback.

${ }^{+}$Mean \pm SD, ${ }^{*} p<0.05$. 
and real-time visual feedback than pre-test of forward head posture group during overhead arm lift test. In individuals with FHP, the upper trapezius, rather than their weakened cervical extensor muscles, supports the weight of the head. ${ }^{20}$ In addition, Limon's study found that higher activity in the upper trapezius changes the angle of the cervical vertebra. ${ }^{21}$ Therefore, during the overhead arm lift test, the upper trapezius compensates for the cervical extensor muscles and maintains the head position in the forward head posture group but not the control group. Thus, visual feedback is thought to result in reduced upper trapezius activity, as introducing the proper head posture can decrease upper trapezius over-activity.

The results of lower trapezius activity decreased similarly to upper trapezius muscle activity. The postural control such as posture correction and real-time visual feedback reduced lower trapezius muscle activity during overhead arm lift test. These findings likely result from abnormal posture, as forward head posture produces excessive lower trapezius activation and abnormal scapula movement. Kim and $\mathrm{Lee}^{22}$ showed that the muscle activity in the active postural control stimulated proprioception and reduced excessive muscle activity. This study's results are consistent with previous study, as active postural control via visual feedback reduced excessive muscle activity. Therefore, it is believed that during the overhead arm lift test, incorrect patterns of scapular motion were corrected through visual feedback, reducing lower trapezius activity.

Sternocleidomastoid muscle activity significantly reduced realtime visual feedback and control group than pre-test. FHP is associated with weakness in the deep cervical flexors and shortening of the opposing cervical extensors. ${ }^{8}$ It also decreases activation of the deep cervical flexors, which is related to increased activation in the superficial muscles, such as the sternocleidomastoid, during craniocervical flexion tasks. ${ }^{23}$ Therefore, the high sternocleidomastoid activity of the forward head posture group resulted from neck instability and FHP. The good alignment of head using real-time visual feedback prevented excessive activity for superficial neck flexor and made the head position to be maintained during overhead arm lift test.

After being subjected to the posture correction and visual feedback conditions, serratus anterior activity increased, but not significantly. However, visual feedback condition values were similar to those of the control group.

Serratus anterior activity can improve scapular stability and assist with scapular upward rotation to allow subjects to perform the overhead arm lift test. Therefore, it is that during the overhead arm lift test the serratus anterior muscle is not directly involved in the stability of the neck but has a stabilizing role for the movement of the arm thus serratus anterior muscle activity was not significantly. Therefore, visual feedback can be a useful way to facilitate serratus anterior activity during the test.

Based on these results, providing visual feedback during the overhead arm lift test appeared to create correct posture, thus ensuring cervical stability. Upper trapezius, sternocleidomastoid, and lower trapezius activity appeared to contribute to scapula movement and cervical stability through decreasing muscle activity, which occurred more significantly in the visual feedback condition than in the pre-test condition. As a result, head position changes decreased during the overhead arm lift test.

The muscle activities of upper trapezius, lower trapezius, serratus anterior, and sternocleidomastoid in real-time visual feedback condition showed little difference from the control group. Also the realtime visual feedback in forward head posture group reduced the distance of head position to the level close to that of the control group. These results mean that head posture control using real-time visual feedback in subjects with forward head posture resemble that seen in the normal subjects during overhead arm lift test. Therefore, the overhead arm lift test can confirm the neck instability of the forward head posture group members, and neck stability can be ensured by controlling posture.

The limitations of this study are that it is difficult to generalize to all age groups because subjects of various ages could not be recruited. It is also difficult to confirm the effects of the short intervention period. Therefore, in future studies, it will be necessary to determine whether or not a subject's posture can be changed in this way by designing a study with a longer intervention period that includes subjects of various ages.

\section{REFERENCES}

1. Cagnie B, Cools A, Deloose V et al. Differences in isometric neck muscle strength between healthy controls and women with chronic neck pain: the use of a reliable measurement. Arch Phys Med Rehabil. 2007;88(11): 1441-5.

2. Kim IG, Lee SY. The Effect of forward head posture and tension type headache on neck movement: for office worker. J Kor Phys Ther. 2018; 30(4):108-11.

3. Yoo WG, Yi CH, Cho SH et al. Effects of the height of ball-backrest on 
head and shoulder posture and trunk muscle activity in VDT workers. Ind Health. 2008;46(3):289-97.

4. Chiu TT, Ku WY, Lee MH et al. A study on the prevalence of and risk factors for neck pain among university academic staff in Hong Kong. J Occup Rehabil. 2002;12:77-91.

5. Hanten WP, Lucio RM, Russell JL et al. Assessment of total head excursion and resting head posture. Arch Phys Med Rehabil. 1991;72(11): $877-80$.

6. Lee KJ, Han HY, Cheon SH et al. The effect of forward head posture on muscle activity during neck protraction and retraction. J Phys Ther Sci. 2015;27(3):977.

7. Kendall FP, McCreary EK, Provance PG et al. Muscles: testing and function, with posture and pain. 5th edition, Philadelphia, LLW, 2014.

8. Griegel-Morris P, Larson K, Mueller-Klaus K et al. Incidence of common postural abnormalities in the cervical, shoulder, and thoracic regions and their association with pain in two age groups of healthy subjects. Phys Ther. 1992;72(6):425-31.

9. Quek J, Pua YH, Clark RA et al. Effects of thoracic kyphosis and forward head posture on cervical range of motion in older adults. Man Ther. 2013;18(1):65-71.

10. Falla D, Jull G, Russell T et al. Effect of neck exercise on sitting posture in patients with chronic neck pain. Phys Ther. 2007;87(4):408-17.

11. Edmondston SJ, Sharp M, Symes A et al. Changes in mechanical load and extensor muscle activity in the cervico-thoracic spine induced by sitting posture modification. Ergonomics. 2011;54(2):179-86.

12. Comerford M, Mottram S. Kinetic control: the management of uncontrolled movement, Chatswood, Elsevier Australia, 2012.

13. Koritnik T, Koenig A, Bajd T et al. Comparison of visual and haptic feedback during training of lower extremities. Gait Posture. 2010;32(4):540-6.
14. Weon JH, Kwon OY, Cynn HS et al. Real-time visual feedback can be used to activate scapular upward rotators in people with scapular winging: an experimental study. J Physiother. 2011;57(2):101-7.

15. Salahzadeh Z, Maroufi N, Ahmadi A et al. Assessment of forward head posture in females: observational and photogrammetry methods. J Back Musculoskelet Rehabil. 2014;27(2):131-9.

16. Yip CH, Chiu TT, Poon AT. The relationship between head posture and severity and disability of patients with neck pain. Man Ther. 2008;13(2): 148-54.

17. Fernandez-de-las-Penas C, Alonso-Blanco C, Cuadrado ML et al. Forward head posture and neck mobility in chronic tension-type headache: a blinded, controlled study. Cephalalgia. 2006;26(3):314-9.

18. Cram J, Kasman G, Holtz J. Introduction to surface electromyography. Gaithersburg, Aspen publishers Inc., 1998.

19. Schafer RC. Clinical biomechanics: musculoskeletal actions and reactions, Philadelphia, Williams \& Wilkins, 1987.

20. Neumann DA. Kinesiology of the musculoskeletal system: foundations for rehabilitation, 2nd ed, St. Louis, Mosby, 2010.

21. Limon S, Valinsky LJ, BenShalom Y. Children at risk: risk factors for low back pain in the elementary school environment. Spine. 2004;29(6):697702.

22. Kim YN, Lee DK. Effects of active vibration exercise on neck pain, disability index, and muscle activity of patients with forward head posture. J Kor Phys Ther. 2018;30(6):218-23.

23. Madeleine P, Mathiassen SE, Arendt-Nielsen L. Changes in the degree of motor variability associated with experimental and chronic neck-shoulder pain during a standardized repetitive arm movement. Exp Brain Res. 2008;185(4):689-98. 